\title{
artigo
}

Costa, E.S.; Medeiros, R.R.P.; Pereira da Silva, J.R.; Pereira da Silva, A.; Carvalho, L.F.F.; Sousa da Silva, G.N.;

Análise da importância do plano de parto na assistência de enfermagem: revisão integrativa

\section{Análise da importância do plano de parto na assistência de enfermagem: revisão integrativa}

\author{
Analysis of the importance of the labor plan in nursing care: integrative review
}

Análisis de la importancia del plan laboral en la atención de enfermería: revisión integrativa

\begin{abstract}
RESUMO
Objetivo:Este estudo teve por objetivo geral analisar a importância do plano de parto na assistência de enfermagem.Método:Estudo de revisão integrativa, na modalidade descritivo-exploratório, com abordagem qualitativa. Este trabalho utilizou de bases de dados para sua construção, que foram SciELO e LILACS com publicações científicas no período de 2015 a 2020, mediante critérios de inclusão e exclusão, incluindo 4 publicações. Resultados:Fica esclarecido o quão importante é o plano de parto para a mulher durante a assistência de enfermagem, por propiciar maior conhecimento sobre o seu parto, que é um momento tão singular para a ela, e proporcionar a humanização na assistência à saúde materno-infantil. Conclusão: Os profissionais de saúde da atenção primária exercem um papel fundamental, principalmente o enfermeiro na realização do pré-natal, que pode construir o plano de parto com a gestante, auxiliando na informatização do parto e fortalecendo a comunicação junto a maternidade. DESCRITORES: Parto; Enfermagem; Assistência.
\end{abstract}

\section{ABSTRACT}

Objective:This study aimed to analyze the importance of the birth plan in nursing care. Integrative review study, in the descriptive-exploratory modality, with a qualitative approach. Method: This work used databases for its construction, which were SciELO and LILACS with scientific publications in the period from 2015 to 2020, using inclusion and exclusion criteria, including 4 publications. Result; It is clear how important the birth plan is for women during nursing care, as it provides greater knowledge about their delivery, which is such a unique moment for them, and provides humanization in maternal and child health care. Conclusion:Primary care health professionals play a fundamental role, especially the nurse in carrying out prenatal care, who can build the birth plan with the pregnant woman, assisting in the computerization of childbirth and strengthening communication with the maternity.

DESCRIPTORS: Childbirth; Nursing; Assistance.

\section{RESUMEN}

Objetivo:Este estudio tuvo como objetivo analizar la importancia del plan de parto en la atención de enfermería. Método: Estudio de revisión integradora, en la modalidad descriptiva-exploratoria, con abordaje cualitativo. Este trabajo utilizó para su construcción bases de datos, las cuales fueron SciELO y LILACS con publicaciones científicas en el período de 2015 a 2020, utilizando criterios de inclusión y exclusión, incluyendo 4 publicaciones. Resultado:Es evidente la importancia que tiene el plan de parto para la mujer durante el cuidado de enfermería, ya que proporciona un mayor conocimiento sobre su parto, que es un momento único para ellas, y humaniza la atención de la salud maternoinfantil. Conclusión: Los profesionales de la salud de atención primaria juegan un papel fundamental, especialmente la enfermera en la realización de la atención prenatal, quienes pueden construir el plan de parto con la gestante, asistiendo en la informatización del parto y fortaleciendo la comunicación con la maternidad.

DESCRIPTORES: Parto; Enfermería; Asistencia.

RECEBIDO EM: 28/09/2020 APROVADO EM: 10/11/2020

\section{Erlânia Souza Costa}

Enfermeira. Pós-graduada em obstetrícia e neonatologia.

ORCID: 0000-0002-5018-0404

\section{Rosicleide Rúbia Pereira Medeiros}

Enfermeira. Pós-graduada em obstetrícia e neonatologia. Mestranda no programa de pós-graduação em educação, trabalho e inovação em medicina na Universidade Federal do Rio Grande do Norte.

ORCID: 0000-0002-3442-7999 


\section{Janine Rosaline Pereira da Silva}

Enfermeira. Pós-graduada em obstetrícia e neonatologia.

ORCID: 0000-0002-2555-3299

\section{Aline Pereira da Silva}

Enfermeira. Pós-graduada em obstetrícia e neonatologia.

ORCID: 0000-0001-8185-9471

\section{Luciane de Fátima Fernandes de Carvalho}

Enfermeira. Pós-graduanda em obstetrícia e neonatologia.

ORCID: 0000-0002-2484-6129

\section{Glaydes Nely Sousa da Silva}

Enfermeira. Pós-graduada em Unidade de Terapia Intensiva. Mestre em Saúde da Família pela Faculdade de Enfermagem Nova Esperança.

ORCID: 0000-0002-6564-657X

\section{INTRODUÇÃO}

$\mathbf{0}$ parto é um processo fisiológico, um momento de vulnerabilidade da mulher, onde tudo ao seu redor passar a ter sentido e influenciar em seu futuro $^{(1)}$. O parto e o pré-natal caminham juntos, o acontecimento de um é o resultado do outro, é preciso garantir a continuidade do cuidado para a gestante, partindo da atenção primária para a atenção secundária e terciária.

Nesse contexto, o profissional que atende a mulher, precisa ter o conhecimento científico para oferecer a melhor assistência possível para a mesma, tornando o momento do parto verdadeiramente especial $^{(2)}$.

Continuamente, deve-se considerar que a atenção obstétrica e neonatal tem predominantes a atuação mediante o modelo biomédico de saúde, que muitas vezes não utiliza tecnologias para qualificar o cuidado, voltando-se para práticas rotineiras, dependente de tecnologias complexas e direcionado para um único profissional, o médico ${ }^{(2)}$.

Sheila Kitzinger em 1980, nos Estados unidos, descreveu a definição do Plano de Parto (PP) $)^{(3)}$. "Um Plano de Parto e Nascimento é um documento escrito, de caráter legal, em que a mulher grávida, recebe informações sobre a gravidez e o processo de parto, considerando seus valores e desejos pessoais"(3).

O plano de parto contém todas as ne- cessidades de mulher, e tem espaço para acrescentar aquilo que seja de sua vontade, o PP trabalha desde o acompanhante escolhido pela mulher, até a quem irá realizar os cuidados com o $\mathrm{RN}^{(3)}$.

...deve-se considerar

que a atenção

obstétrica e neonatal tem predominantes a atuação mediante o modelo biomédico de saúde...

Com base nesse conceito, os enfermeiros e demais profissionais de saúde habilitados, devem utilizar em suas consultas este documento, buscando melhorar a assistência e fortalecer a comunicação da mulher gestante no ambiente hospitalar. Além disso, é um instrumento de empoderamento feminino, buscando conhecer os desejos e necessidades da mulher naquele momento tão singular que é a gestação ${ }^{(4)}$.

Percebe-se a relevância de se realizar pesquisas voltadas a essa temática, sabendo que resultará em qualificação do cuidado e humanização no pré-natal. Sendo assim, levantou-se o seguinte questionamento: Qual a importância do plano de parto na assistência de enfermagem encontrada nas publicações científicas atuais? Desse modo, este estudo teve por objetivo geral: analisar a importância do plano de parto na assistência de enfermagem.

\section{MÉTODO}

Estudo de revisão integrativa, na modalidade descritivo-exploratório, com abordagem qualitativa. Este trabalho utilizou de bases de dados para sua construção, que foram Scientific Electronic Library Online (SciELO) e Literatura Latino-americana e do Caribe em Ciências da Saúde (LILACS), que foram selecionadas mediante critérios de inclusão e exclusão. A coleta de dados ocorreu no mês de fevereiro de 2020. Este estudo foi guiado pelo instrumento PRISMA.

Esta pesquisa teve como critérios de inclusão: artigos em português, inglês ou espanhol, na modalidade original ou revisão integrativa, no período temporal esta- 
belecido de 2015 a 2020, publicados integralmente e disponíveis gratuitamente nas bases de dados selecionadas. Foram excluídos da pesquisa, todas as publicações fora do período estabelecido, na modalidade de estudo de caso, relatos de experiência e estudos de caso controle.

$\mathrm{Na}$ primeira busca na base de dados da SciELO, utilizou-se os três descritores: "Parto" AND "Enfermagem" AND "Assistência. No primeiro momento foram encontradas 903 publicações. Após a aplicação do filtro de tempo, idioma e modalidade da pesquisa, ficaram $185 \mathrm{pu}-$ blicações. Foi realizada a leitura dos títulos e dos resumos das 185 publicaçóes, e 184 publicações não correspondiam sobre o tema, restando apenas 1 publicação. A única publicação que estava dentro dos critérios de inclusão foi analisada integralmente e inserida no corpo da pesquisa.

Foi realizada uma segunda busca na mesma base de dados, utilizando os descritores "Plano de parto" AND "Enfermagem" Inicialmente foram encontradas 8 publicações. Aplicando o filtro de tempo, idioma permaneceram as 8 publicações. Em seguida realizei a leitura dos títulos e resumos, onde foram eliminadas 7 publicações, por não estarem dentro dos critérios de inclusão da pesquisa. Desse modo na segunda busca restou apenas 1 publicação. Sendo assim, o total de publicações encontradas na base de dados da SciELO foram de 2 publicações.

O descritor "Plano de parto" não está diretamente cadastrado nos descritores de ciências da saúde (Decs), mas foi utilizado como termo chave desta pesquisa, para consolidar as publicações a serem encontradas, visto que descritor "Parto" é um termo generalista e trouxe diversas publicações relacionadas ao parto, mas não a temática de forma específica.

$\mathrm{Na}$ segunda base de dados, a LILACS, em sua primeira busca, foram usados três descritores: "Parto" AND "Enfermagem" AND "Assistência. No primeiro momento foram encontradas 265 publicações, após a aplicação do filtro de tempo, idioma e modalidade da pesquisa, ficaram 80 publicações. Foi realizada a leitura dos títulos e dos resumos das 80 publicaçóes, e restaram apenas 3 publicações. Dessas 3 publicações, 1 publicação obteve duplicidade, e 2 publicações estavam dentro dos critérios de inclusão, foram analisadas integralmente e inserida no corpo da pesquisa.

Foi feita uma segunda busca na mesma base de dados, utilizando os descritores "Plano de parto" AND "Enfermagem". Nesta segunda busca não obtive nenhum resultado, permanecendo 2 publicações da base LILACS no corpo da pesquisa. Por fim, foram incluídos nesta pesquisa o total de 4 publicações acerca de temática escolhida, unificando as duas bases de dados.

Após a análise das publicações, elas foram organizadas em um quadro,utilizando o programa da microsoft WORD, contendo as informações mais pertinentes sobre a temática, tais como: autores da publicação, ano, periódico, título das publicações e os resultados das publicações que se referem a importância do plano de parto na assistência de enfermagem.

\section{RESULTADOS}

\section{Quadro 1- Apresentação das publicações científicas sobre o plano de parto na assistência de enfermagem, João} Pessoa, Paraíba, 2020.

\begin{tabular}{|c|c|c|c|c|}
\hline $\begin{array}{l}\text { AUTORES DAS } \\
\text { PUBLICAÇÕES }\end{array}$ & TÍTULO DAS PUBLICAÇÕES & ANO & PERIÓDICO & $\begin{array}{l}\text { A IMPORTÂNCIA DO PLANO DE PARTO NA } \\
\text { ASSISTÊNCIA DE ENFERMAGEM. }\end{array}$ \\
\hline $\begin{array}{l}\text { Lopezosa PH, } \\
\text { Maestre MH e } \\
\text { Borrego MAR. }\end{array}$ & $\begin{array}{c}\text { O cumprimento do plano } \\
\text { de parto e sua relação com } \\
\text { os resultados maternos e } \\
\text { neonatais. }\end{array}$ & 2017. & $\begin{array}{l}\text { Rev. Latino Americana } \\
\text { de Enfermagem. }\end{array}$ & $\begin{array}{l}\text { - Em muitos locais o plano de parto não é realizado. } \\
\text { - O artigo traz que à medida que os planos de parto } \\
\text { forem sendo cumpridos, os resultados maternos e } \\
\text { infantis serão melhores. } \\
\text { - No estudo, apenas } 37 \% \text { das mulheres tiveram o } \\
\text { plano de parto cumprido. } \\
\text { - O plano de parto pode favorecer a comunicação e } \\
\text { a prática obstétrica entre os profissionais de saúde. }\end{array}$ \\
\hline $\begin{array}{l}\text { Medeiros RMK, } \\
\text { Figueiredo G, } \\
\text { Correa ACP e } \\
\text { Barbieri M. }\end{array}$ & $\begin{array}{l}\text { Repercussões da utilização } \\
\text { do plano de parto no } \\
\text { processo de parturição. }\end{array}$ & 2019. & $\begin{array}{l}\text { Revista Gaúcha de } \\
\text { Enfermagem. }\end{array}$ & $\begin{array}{l}\text { - O profissional de saúde, que é frequentemente } \\
\text { o enfermeiro, deve preencher o plano de parto } \\
\text { preferencialmente na atenção primária a saúde, } \\
\text { para que em um momento que antecede o parto, } \\
\text { haja comunicação com a maternidade. } \\
\text {-O plano de parto é uma ferramenta educacional } \\
\text { para ensinar as mulheres sobre o parto. } \\
\text { - O enfermeiro apoia e estimula a prática do plano } \\
\text { de parto. }\end{array}$ \\
\hline
\end{tabular}




\begin{tabular}{|c|c|c|c|c|}
\hline $\begin{array}{l}\text { Narchi NZ, Venân- } \\
\text { cio KCMP, Ferreira } \\
\text { FM, e Vieira JR. }\end{array}$ & $\begin{array}{c}\text { O plano individual de } \\
\text { parto como estratégia de } \\
\text { ensino-aprendizagem das } \\
\text { boas práticas de atenção } \\
\text { obstétrica. }\end{array}$ & 2019. & $\begin{array}{l}\text { Revista de Escola de } \\
\text { Enfermagem da USP. }\end{array}$ & $\begin{array}{l}\text {-O plano de parto viabiliza o protagonismo da } \\
\text { mulher. } \\
\text { - O plano de parto permite tratar a mulher de } \\
\text { forma individual, com respeito. }\end{array}$ \\
\hline $\begin{array}{l}\text { Gomes RPC, } \\
\text { Silva RS, Oliveira } \\
\text { DCC, Manzo BF, } \\
\text { Guimarães GL e } \\
\text { Souza KV. }\end{array}$ & $\begin{array}{c}\text { Plano de parto em rodas } \\
\text { de conversa: escolhas das } \\
\text { mulheres. }\end{array}$ & 2017. & $\begin{array}{l}\text { Revista Mineira de } \\
\text { Enfermagem. }\end{array}$ & $\begin{array}{l}\text { - O profissional de saúde tem a responsabilidade } \\
\text { de informar a mulher e auxiliar na tomada de } \\
\text { decisão. } \\
\text {-As mulheres têm o desejo de praticar o que tem } \\
\text { nas informações do plano de parto. } \\
\text {-Cabe aos profissionais da atenção primária a } \\
\text { saúde, informar a mulher e auxiliar na construção } \\
\text { do seu plano de parto desde o pré-natal. }\end{array}$ \\
\hline
\end{tabular}

Nos resultados das publicações, fica esclarecido, o quão importante é o plano de parto para a mulher durante a assistência de enfermagem, por propiciar maior conhecimento sobre o seu parto, que é um momento tão singular para a ela, e proporcionar a humanização na assistência à saúde materno-infantil.

Foi possível perceber que os profissionais de saúde da atenção primária exercem um papel fundamental, principalmente o enfermeiro, na realização do pré-natal, que pode construir o plano de parto junto com a gestante, auxiliando na informatização do parto e fortalecendo a comunicação junto a maternidade.

\section{DISCUSSÃO}

O processo de parto e sua assistência perpassou por mudanças relevantes ao longo dos anos. Inicialmente, dava-se a ênfase às tecnologias duras ou leve duras para auxiliar no parto, além de ter o envolvimento de todo um contexto tradicional, composto por parteiras, ambiente familiar e íntimo da mulher. Logo em seguida, o processo de parir se direcionou para o ambiente cirúrgico ${ }^{(5)}$.

Continuamente, esse modelo de assistência contribuiu para uma perda significativa da autonomia da mulher, criando um déficit em sua participação no trabalho de parto e parto, trazendo um forte

\section{Inicialmente,}

dava-se a ênfase

às tecnologias

duras ou leve duras

para auxiliar no

parto, além de ter

o envolvimento de

todo um contexto

tradicional,

composto por

parteiras, ambiente

familiar e íntimo

da mulher. impacto no nascimento, onde a mulher deixa de ser protagonista de um momento em que ela é a autora principal ${ }^{(6)}$.

O PP é um documento escrito, legalizado, que demonstra os desejos das mulheres e suas expectativas para o trabalho de parto e parto, podendo ser acrescentando o período puerperal imediato, levando em consideração os princípios e valores da mulher ${ }^{(7,8)}$. Vale ressaltar, que o PP é ampliado, pois ele inclui os desejos da mulher relacionado ao acompanhante, a suas escolhas alimentares, posições para o parto, cuidados ao recém-nascido, dentre outras características $^{(9)}$.

Com base nesse contexto, o PP, após sua construção durante o pré-natal, deve ser encaminhado junto com a gestante e apresentado a equipe da maternidade que cuidará daquela mulher. É interessante que os profissionais da atenção primária a saúde, principalmente o enfermeiro, realize a construção deste plano de parto, e façam a mediação, para que estas pacientes alcancem o serviço de saúde secundário com efetividade ${ }^{(8)}$.

O momento do nascimento por muito tempo, era considerado exclusivo das mulheres, não se comentava em um parto com a presença de acompanhante, familiares, ou quaisquer que fosse a pessoa para auxiliar naquele processo, o parto era visto como um momento de força, poder e coragem da mulher, apenas dela ${ }^{(10)}$. 
Com isso, o PP vem para garantir a autonomia da mulher, fortalecendo essa força que o momento do parto já traz consigo, mas garantindo o seu direito, levando em consideração a singularidade e peculiaridades de cada mulher, para que elas possam tomar suas decisóes e opinar para um momento único em suas vidas ${ }^{(3)}$.

É o PP que dá a voz as gestantes, garante a segurança da mulher, rompe com as fragilidades do cuidado, organiza as informações da mulher, considera sua cultura, seus medos e anseios ${ }^{(11)}$. Implantar o PP no pré-natal é de extrema importância, porém, é preciso analisar o conhecimento do enfermeiro quanto a isso, estimulando a afetividade deste profissional a este momento tão importante ${ }^{(12)}$.

\section{CONCLUSÕES}

Com isso, o PP vem para garantir

a autonomia

da mulher,

fortalecendo

essa força que o

momento do parto

já traz consigo...
A partir do plano de parto a mulher pode conhecer sobre o seu próprio parto, e se fortalecer acerca das informações que constam dentro dele. $\mathrm{O}$ enfermeiro e sua equipe, devem estimular a construção do plano de parto desde o pré-natal, buscando orientar a mulher e a família sobre o todos os procedimentos que irão acontecer com ela e com o bebê.

É preciso incentivar os profissionais a realizar novas pesquisas e transformar a sua prática em saúde, visando humanizar a assistência e propiciar ao binômio o que há de melhor diante do cuidado integral. O plano de parto proporciona a comunicação entre os serviços de saúde primária, secundária e terciária, e isso é de extrema relevância. -

\section{REFERÊNCIAS}

1. Barros APZ. de, Lipinski JM, Sehnem GD, Rodrigues AN, Zambiazi ES da. The Knowledge Of Nurses About The Delivery Plan. Rev Enferm. UFSM. [internet] 2017 [cited 2020 abr 2] Jan/Fev.;7(1): 69-79. Available from: https://periodicos.ufsm.br/reufsm/article/ view/23270/pdf

2. Brasil. Ministério da Saúde. Cadernos Humaniza SUS. [internet]. Brasília (DF): Ministério da Saúde; 2014 [cited 2020 abr 5]. (Humanização do Parto e do Nascimento. Available from: http:// bvsms.saude.gov.br/bvs/publicacoes/cadernos_humanizaSUS. pdf.

3. Suárez MC, Barranco DA, Jordana MC, Roche MEM. Uso e influência dos planos de parto e nascimento no processo de parto humanizado. Rev Latinoam Enferm. [internet] 2015 [cited 2020 mar 23];23(3):520-6. Available from: http://www.scielo.br/pdf/ rlae/2015nahead/pt_0104-1169-rlae-0067-2583.pdf .

4. Silva Alnv da, Neves AB das, Sgarbi AKG, Souza RA. Plano De Parto: Ferramenta para o Empoderamento de Mulheres Durante a Assistência de Enfermagem. Rev Enferm UFSM. [internet] 2017 [cited 2020 mar 15] Jan/Fev.; 7(1): 144-151. Available from: https://periodicos.ufsm.br/reufsm/article/view/22531/pdf.

5. Leão MRDC, Riesco MLG, Schneck CA, Angelo M. Reflexões sobre o excesso de cesarianas no Brasil e a autonomia das mulheres. Cienc Saúde Coletiva. [internet] 2013; [cited 2020 abr 21] 18(8):2395-400. Available from: https://https:/.doi.org/10.1590/ S1413-81232013000800024.

6. Mouta, RJO, Silva TMA de, de Melo PTS de, Lopes NS de, Moreira VA dos. Plano de parto como estratégia de empoderamento feminino. Rev Baiana Enferm. [internet] 2017 [cited 2020 abr 25];31(4):e20275. Available from doi: https://doi.org/10.18471/ rbe.v31i4.20275.
7. Aragon $\mathrm{M}$ et al. Perspetives of expectante women and health care providers on birth plans. J Obstet Gynaecol Can. [internet] 2013 [cited 2020 abr 28];35(11):979-85. Available from: https:// www.ncbi.nlm.nih.gov/pubmed/24246397.

8. Gomes RPC. Delivery plan in conversation circles: women's choices. Rev Min Enferm. [internet] 2017 [cited 2020 abr 12];21:e1033. Available from: doi: http://www.dx.doi.org/10.5935/14152762.20170043

9. Mei JY,et al. Birth plans: what matters for birth experience satisfaction. Birth. [internet] 2016 [cited 2020 mar 18] jun;43(2):144- 50. Available from::http://www.ncbi.nlm.nih.gov/ pubmed/26915304.

10. Macedo JG, Arraes R. Autonomia da gestante na escolha de parto na realidade da prestação de assistência médico-hospitalar brasileira. In: VII Jornada de Sociologia da Saúde - Saúde como Objeto do Conhecimento: História e Cultura. [internet] Curitiba, Novembro de 2013 [cited 2020 abr 2]. Available from:file://IC:/Users/Mariana/Downloads/9098-Texto\%20do\%20artigo-31660-1-10-20151208\%20(1).pdf

11. Andrezzo HFA. O desafio do direito à autonomia: uma experiência de Plano de Parto no SUS. [Dissertação Mestrado em Ciências]. Universidade de São Paulo, São Paulo, 2016, 111p. Available from: https://www.teses.usp.br/teses/disponiveis/6/6136/tde07112016-141429/pt-br.php.

12. Guerreiro EM, Rodrigues DP, Silveira MAM da, Nájori Bárbara Ferreira de Lucena NBF. Cuidado pré-natal na atenção básica de saúde sob o ollhar de gestantes e enfermeiros. Rev. Min. Enferm. [internet] ;16(3): 315-323, jul./set., 2012 [cited 2020 abr 25]. Available from: file://C:/Users/Mariana/Downloads/v16n3a02. pdf. 